\title{
Role of Primate Substantia Nigra Pars Reticulata in Reward-Oriented Saccadic Eye Movement
}

\author{
Makoto Sato, ${ }^{1,2}$ and Okihide Hikosaka ${ }^{1}$ \\ Departments of ${ }^{1}$ Physiology and ${ }^{2}$ Neurosurgery, Juntendo University, School of Medicine, Tokyo 113-8421, Japan
}

To test the hypothesis that the basal ganglia are related to reward-oriented saccades, we examined activity of substantia nigra pars reticulata ( $\mathrm{SNr}$ ) neurons by using a one-directionrewarded version of the memory-guided saccade task (1DR). Many SNr neurons changed (decreased or increased) their activity after and before a visual cue (post-cue and pre-cue activity). Post-cue decreases or increases tended to be larger to a contralateral cue. They were often modulated prospectively by the presence or absence of reward, either positively (enhanced in the rewarded condition) or negatively (enhanced in the nonrewarded condition). The positive reward modulation was more common among decreasing type neurons, whereas no such preference was observed among increasing type neurons. The reward-contingent decrease in SNr neuronal activity would facilitate rewarded saccades by inducing disinhibition in superior colliculus (SC) neurons. In contrast, the increase in $\mathrm{SNr}$ activity would suppress a saccade less selectively (rewarded or nonrewarded) by augmenting inhibition of SC neurons. The post-cue activity was often preceded by anticipatory pre-cue activity. Most typically, post-cue decrease was preceded by pre-cue decrease, selectively when the contralateral side was rewarded. This would reinforce the reward-oriented nature of $\mathrm{SNr}$ neuronal activity. The decreases and increases in $\mathrm{SNr}$ activity may be derived directly and indirectly, respectively, from the caudate (CD), where neurons show reward-contingent precue and post-cue activity. These results suggest that the CDSNr-SC mechanism would promote saccades oriented to reward.

Keywords: basal ganglia; monkey; single-unit recording; memory-guided saccade; motivation; reward expectation
A major function of the basal ganglia is to control body movements (DeLong and Georgopoulos, 1981). On the other hand, recent studies have implicated the basal ganglia in learning or adaptation of behavior based on reward or motivation (Houk et al., 1995; Schultz, 1998). However, it is still unclear how sensorimotor and motivational functions are integrated in the basal ganglia. A critical step toward a solution of this problem would be to study the final output of the basal ganglia, specifically to examine whether and how the movement- and reward-related signals are integrated as spike activity of single neurons.

The substantia nigra pars reticulata $(\mathrm{SNr})$ is a major output station of the basal ganglia. The $\mathrm{SNr}$ controls saccadic eye movement with its inhibitory connection to the superior colliculus (SC) (Hikosaka and Wurtz, 1989; Hikosaka et al., 2000). Neuronal activity in the $\mathrm{SNr}$ is often selective for behavioral contexts (Hikosaka and Wurtz, 1983b; Handel and Glimcher, 2000), especially when saccades are guided by memory (Hikosaka and Wurtz, $1983 \mathrm{~b})$. These results suggest that $\mathrm{SNr}$ neurons are involved in non-sensorimotor processes, including cognitive and motivational ones.

\footnotetext{
Received Sept. 4, 2001; revised Dec. 26, 2001; accepted Dec. 26, 2001.

This work was supported by a Grant-in-Aid for Scientific Research on Priority Areas (C) of Ministry of Education, Culture, Sports, Science and Technology, Core Research for Evolutional Science and Technology of Japan Science and Technology Corporation, and Japan Society for the Promotion of Science Research for the Future program. We thank Johan Lauwereyns, Shunsuke Kobayashi, Yoriko Takikawa, Reiko Kawagoe, Hiro Nakahara, and Hideaki Itoh for helpf ul comments, Makoto Kato and Brian Coe for designing the computer programs, and Masashi Koizumi for technical support.

Correspondence should be addressed to Okihide Hikosaka, Laboratory of Sensorimotor Research, National Eye Institute, National Institutes of Health, Building 49, Room 2A50, Bethesda, MD 20892. E-mail: oh@1sr.nei.nih.gov.

Copyright (C) 2002 Society for Neuroscience $0270-6474 / 02 / 222363-11 \$ 15.00 / 0$
}

This possibility seems more plausible if we consider the caudate nucleus (CD), which provides the SNr with a major input (Yoshida and Precht, 1971; Hikosaka et al., 1993). CD neurons show visual, mnemonic, and saccadic activity (Hikosaka et al., 1989a,b; Brown et al., 1995; Kermadi and Joseph, 1995) and in addition show reward-predicting activity (Rolls et al., 1983; Nishino et al., 1984; Hikosaka et al., 1989c; Kermadi and Joseph, 1995). Using a one-direction-rewarded (1DR) version of the memory-guided saccade task, a recent study from our laboratory demonstrated integration of visuosaccadic and reward-related signals in single CD neurons (Kawagoe et al., 1998). A simple prediction would then be that $\mathrm{SNr}$ neurons behave similarly to CD neurons. However, there are at least two parallel pathways (direct and indirect) from the CD to the $\mathrm{SNr}$ that are supposed to have opposite actions. Signals carried by $\mathrm{CD}$ neurons as increases in activity would be translated into decreases in activity of $\mathrm{SNr}$ neurons if they are transmitted through the direct $\mathrm{CD}-\mathrm{SNr}$ pathway. In contrast, the same signals would be translated into increases in $\mathrm{SNr}$ neuronal activity if they are transmitted through the indirect pathway (Matsumura et al., 1992; Kato and Hikosaka, 1995). Therefore, $\mathrm{CD}$ neuronal activity alone cannot predict the outcome of the processing in the basal ganglia.

In the present study, we used the same task (1DR) to investigate whether and how visuosaccadic and reward-related signals are integrated in $\mathrm{SNr}$ neurons. We found that $\mathrm{SNr}$ neurons responded to or predicted a visual cue stimulus as $\mathrm{CD}$ neurons do, but by decreasing or increasing their activity. The two types of $\mathrm{SNr}$ neurons were different such that the decreasing activity, not the increasing activity, tended to be enhanced in rewarded trials. 


\section{MATERIALS AND METHODS}

\section{General}

We used two male Japanese monkeys (Macaca fuscata). The monkeys were kept in individual primate cages in an air-conditioned room where food was available ad libitum. At the beginning of each experimental session, they were moved to the experimental room in a primate chair. The monkeys were given restricted amounts of fluid during periods of training and recording. Their body weight and appetite were checked daily. Supplementary water and fruit were provided daily. All surgical and experimental protocols were approved by the Juntendo University Animal Care and Use Committee and are in accordance with the National Institutes of Health Guide for the Care and Use of Animals.

\section{Surgical procedures}

Before the recording experiments started, we implanted a head holder, a chamber for unit recording, and an eye coil under the following surgical procedures. The monkey was sedated with ketamine $(4.6-6.0 \mathrm{mg} / \mathrm{kg}$ ) and xylazine $(1.8-2.4 \mathrm{mg} / \mathrm{kg})$ given intramuscularly, and then general anesthesia was induced by intravenous injection of pentobarbital (4.5-6.0 $\left.\mathrm{mg} \cdot \mathrm{kg}^{-1} \cdot \mathrm{hr}^{-1}\right)$ with butarphanod tartrate $\left(0.02 \mathrm{mg} \cdot \mathrm{kg}^{-1} \cdot \mathrm{hr}^{-1}\right)$. Surgical procedures were performed under aseptic conditions in an operating room. After the skull was exposed, 10-15 acrylic screws were bolted into it. The screws acted as anchors by which a head holder and a chamber, both made of delrin, were fixed to the skull with a dental acrylic resin. The recording chamber, which was rectangular (anteroposterior, $42 \mathrm{~mm}$; lateral, $30 \mathrm{~mm}$; depth, $10 \mathrm{~mm}$ ), was placed over the frontoparietal cortices, tilted laterally by $35^{\circ}$ in the coronal plane, and aimed at the $\mathrm{SNr}$ based on the atlas of Macaca fuscata (Kusama and Mabuchi, 1970). A scleral eye coil was implanted in one eye for monitoring eye position (Robinson, 1963; Judge et al., 1980). The monkey received antibiotics (sodium ampicillin, $25-40 \mathrm{mg} / \mathrm{kg}$, i.m., each day) after the operation.

\section{Behavioral tasks}

The monkey sat in a primate chair in a dimly lit and sound-attenuated room with its head fixed. In front of the monkey was a tangent screen (30 $\mathrm{cm}$ from its face) onto which small red spots of light (diameter, $0.2^{\circ}$ ) were backprojected using two LED projectors. The first projector was used for a fixation point, and the second for an instruction cue stimulus. The position of the cue stimulus was controlled by reflecting the light off two orthogonal (horizontal and vertical) mirrors driven by galvanometers under computer control.

The monkeys were first trained to perform memory-guided saccades (Hikosaka and Wurtz, 1983b) in an all directions-rewarded condition (ADR) (see Fig. 1). In ADR, every correct saccade was rewarded with a drop of water together with the tone stimulus. A task trial started with the onset of a central fixation point on which the monkeys had to fixate. A cue stimulus (spot of light; duration, $100 \mathrm{msec}$ ) came on $1 \mathrm{sec}$ after onset of the fixation point, and the monkeys had to remember its location. After $1-1.5 \mathrm{sec}$, the fixation point turned off, and the monkeys were required to make a saccade to the previously cued location. The target came on 400 msec later for $150 \mathrm{msec}$ at the cued location. The saccade was judged to be correct if the eye position was within a window around the target (usually within $\pm 3^{\circ}$ ) when the target turned off. The monkeys made the saccade before target onset based on memory, because otherwise the eyes could not reach the target window within the $150 \mathrm{msec}$ target-on period; the target was presented only to give the monkeys the accurate feedback information. The next trial started after an intertrial interval of $3.5-4 \mathrm{sec}$.

The monkeys were then trained to perform the memory-guided saccade task in the one-direction-rewarded condition (1DR) (Kawagoe et al., 1998) (see Fig. 1). In 1DR, only one of two directions was rewarded within a block that included 40 successful trials. The rewarded direction was changed in the next block. In some experiments, 1DR was performed with four target directions, among which only one direction was rewarded. Even for the nonrewarded direction, the monkeys had to make a correct saccade. If the saccade was incorrect, the same trial was repeated. The amount of reward per block was set approximately the same between 1DR and ADR; the amount of reward per trial in 1DR was approximately twice that in ADR. Other than the actual reward, no indication was given to the monkeys as to which direction was currently rewarded.

\section{Experimental procedures}

The cue stimulus was presented at one of two positions with the same eccentricity such that one of them was in the response field of the neuron.
They were placed in symmetrical positions with respect to the fixation point. In more than half the cases, the targets were placed horizontally to the right and the left (see Fig. 1). The target eccentricity was 5, 10, or $20^{\circ}$.

Once an $\mathrm{SNr}$ neuron was isolated, the monkeys performed ADR and 1DR. ADR was performed in one block. 1DR was performed in two blocks, each with a different rewarded direction. The order of blocks was randomized for different neurons. We sometimes repeated the 1DR blocks to confirm the reproducibility of the behavior of neuron.

In one block of ADR or 1DR, the target cue was chosen pseudorandomly for each trial such that every sub-block of four trials contained two trials for each target. One block of ADR or 1DR contained 40 successful trials (i.e., 20 trials for each target). In the four-direction version, one block of 1DR or ADR contained 60 successful trials (i.e., 15 trials for each target).

\section{Recording procedures}

Before the single-unit recording experiment, we obtained magnetic resonance images (Hitachi, AIRIS, 0.3 Tesla) such that they were perpendicular to the recording chamber. We then determined the recording sites in the $\mathrm{SNr}$ on the basis of the chamber-based coordinates.

Single-unit recordings were performed using tungsten electrodes (diameter, $0.25 \mathrm{~mm}$; $1-5 \mathrm{M} \Omega$; measured at $1 \mathrm{KHz}$; Frederick Haer). The electrode was inserted into the brain through a stainless steel guide tube (diameter, $0.8 \mathrm{~mm}$ ) that was used to penetrate the dura. Once the location of the SNr was identified, we sometimes implanted a plastic guide tube (diameter, $0.8 \mathrm{~mm}$ ) semipermanently. This procedure, if the guide tube was placed successfully, yielded efficient sampling of $\mathrm{SNr}$ neurons for 10-15 tracks. The guide tube could then be replaced with a new one that was aimed at a different region in the SNr. A hydraulic microdrive (Narishige, MO 95-S) was used to advance the electrode into the brain.

Eye movements were recorded using the search coil method (Enzanshi Kogyo MEL-20U) (Robinson, 1963; Judge et al., 1980; Matsumura et al., 1992). Eye positions were sampled at $500 \mathrm{~Hz}$. The behavioral tasks as well as storage and display of data were controlled by a computer (PC 9801RA, NEC, Tokyo, Japan). The unitary action potentials were passed through a window discriminator (Bak INC, Model DDIS-1), and the times of their occurrences were stored with a resolution of $1 \mathrm{msec}$.

\section{Data analysis}

\section{Pre-cue activity}

For each trial we calculated the firing rates in a test period of $300 \mathrm{msec}$ duration (starting from $200 \mathrm{msec}$ before cue onset) and in a control period of $1000 \mathrm{msec}$ duration (starting from $1300 \mathrm{msec}$ before fixation point onset). We then compared the firing rates between the test and control windows for each block using paired $t$ test. If the difference was statistically significant $(p<0.05)$, it was judged that pre-cue activity was present.

The pre-cue activity thus detected was then classified into decreasing and increasing type if the firing rate was lower or higher, respectively, in the test window than in the control window. The selectivity of the pre-cue activity for the rewarded direction was judged to be present if the magnitudes of the pre-cue activity were different between the two blocks of 1DR using $t$ test $(p<0.05)$.

\section{Post-cue activity}

Determination of the presence of post-cue activity. In the present study we focused on phasic changes in activity after cue onset that would be regarded as visual responses. The presence of post-cue activity was determined by comparing the firing rate in the post-cue test period $(100-200 \mathrm{msec}$ after cue onset) with the firing rate in the control period for individual trials using paired $t$ test. The test was repeated for two kinds of control period (duration, $100 \mathrm{msec}$ ): before fixation point onset and before cue onset. This was because some neurons showed pre-cue activity that often masked post-cue activity. This analysis was done for each of four conditions of 1DR (two cue directions $\times$ two reward conditions). If the result of the paired $t$ test was significant $(p<0.05)$ for any of the conditions, it was determined that the post-cue activity was present.

Classification based on direction and reward modulations. We first determined the preferred direction/reward condition of the neuron (e.g., contralateral/rewarded for the neuron in Fig. 2) by the procedure described below. To test the direction selectivity, the preferred condition was compared with the condition in which the cue direction was opposite and the reward condition was the same (e.g., comparison within the left 
column for the neuron in Fig. 2) using $t$ test $(p<0.05)$. To test the reward selectivity, the preferred condition was compared with the condition in which the cue direction was the same and the reward condition was opposite (e.g., comparison within the top row for the neuron in Fig. $2)$ using $t$ test $(p<0.05)$. On the basis of these analyses, the neuron was classified into four types: direction-only (D-only), reward-only (R-only), direction and Reward (D\&R), and nonmodulated (non-mod).

We then determined for each neuron the kinds of direction and reward modulations. For the direction modulation, neurons of the D-only or D\&R type were divided into contralateral- and ipsilateral-preferring groups, whereas neurons of the R-only or non-mod type were designated to be nonselective (see Table 2). For the reward modulation, neurons of the R-only or D\&R type were divided into positive (reward-preferring) and negative (nonreward-preferring) groups, whereas neurons of the D-only or non-mod type were designated to be nonmodulated (see Table 3).

Determination of the preferred condition of the neuron. For each neuron, we first calculated the normalized response (NR) for each of four conditions of 1DR (two cue directions $\times$ two reward conditions) by the following equation: $\mathrm{NR}=F t / \mathrm{Fc}$, with $F t$ the mean firing rate in the test window and $F c$ the mean firing rate in the control window.

NRs for four conditions were thus obtained: $N R(C-c u e / R+)$ : rewarded contralateral cue; NR(C-cue/R-): nonrewarded contralateral cue; NR(I-cue/R+): rewarded ipsilateral cue; and NR(I-cue/R-): nonrewarded ipsilateral cue.

We determined the preferred condition of the neuron as the one for which the absolute value of $\log (\mathrm{NR})$ was the largest. According to this method, a $2 \times$ increase would be equivalent to one-half decrease of firing rate.

Classification into decreasing/increasing types. Post-cue activity was classified into decreasing or increasing type based on NR for the preferred condition of the neuron(see Tables $1-3$ ). If $\mathrm{NR}<1$ (i.e., $F t<F c$ ), the neuron was defined to be of a decreasing type; if $N R>1$, it was defined to be an increasing type.

Direction and reward indices. Direction index (D-index) and reward index (R-index) were calculated with reference to the preferred condition of the neuron. For D-index, the preferred condition was compared with the condition in which the cue direction was opposite and the reward condition was the same. For R-index, the preferred condition was compared with the condition in which the cue direction was the same and the reward condition was opposite. For example, for a neuron preferring the rewarded contralateral cue $(\mathrm{C}$-cue $/ \mathrm{R}+)$ : $\mathrm{D}$-index $=[\mathrm{NR}(\mathrm{C}$-cue $/ \mathrm{R}+)-$ $\mathrm{NR}(\mathrm{I}$-cue $/ \mathrm{R}+)] /[\mathrm{NR}(\mathrm{C}$-cue $/ \mathrm{R}+)+\mathrm{NR}(\mathrm{I}$-cue $/ \mathrm{R}+)]$; R-index $=[\mathrm{NR}(\mathrm{C}-$ cue/R+) - NR(C-cue/R-)]/[NR(C-cue/R+) + NR(C-cue/R-)].

Note, however, that the signs of these indices would be opposite between decreasing and increasing type neurons; for example, a larger contralateral response would show up as a larger minus value of $\mathrm{D}$-index for a decreasing type neuron but as a larger plus value of D-index for an increasing type neuron. To make the meanings of the indices consistent across the decreasing and increasing types, we reversed the signs of these indices only for the decreasing type. As a consequence, a larger plus value of D-index would indicate a stronger contralateral preference (i.e., a larger decrease or increase of activity in response to a contralateral cue than to an ipsilateral cue), whereas a larger plus value of R-index would indicate a stronger positive reward modulation (i.e., a larger decrease or increase in rewarded trials than in nonrewarded trials).

\section{Histology}

After recording was completed, the monkeys were anesthetized with an overdose of pentobarbital sodium and perfused through the heart with $4 \%$ paraformaldehyde. The brain was blocked and equilibrated with $20 \%$ sucrose. Frozen sections were cut at $50 \mu \mathrm{m}$ in the planes parallel to the electrode penetrations so that complete tracks were visible in single sections. The sections were stained with cresyl violet. Reconstruction of the location and extent of the SNr was based on microlesions $(5 \mu \mathrm{A}$ for $200 \mathrm{sec}$ ) made at the end of some recording experiments. Individual recording and injection sites were estimated on the basis of these microlesions.

\section{RESULTS}

We recorded single-unit activity from the $\mathrm{SNr}$ in three hemispheres of two monkeys. We searched for the SNr using the somatosensory, visual, and auditory nuclei of the thalamus as landmarks. SNr neurons were characterized by their tonic and rapid spontaneous activity $(21-131 \mathrm{~Hz}$; mean, 62.3 Hz) (Hikosaka

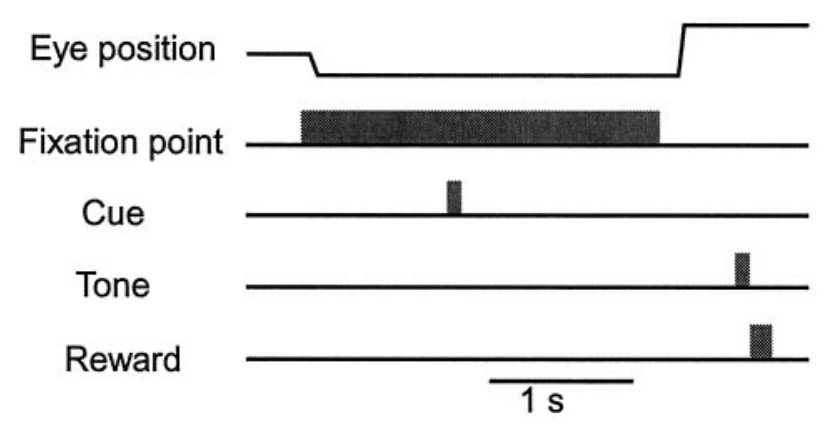

1DR

ADR

\section{contra reward ipsi reward}
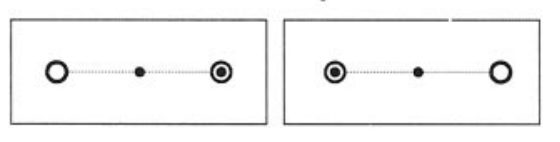

\section{- rewarded o non-rewarded}

Figure 1. Memory-guided saccade task in the "one-direction-rewarded" condition (1DR) and "all-directions-rewarded" condition (ADR). Top, Timing of stimulus presentation and eye movements. Bottom, An experiment consisted of two blocks of 1DR task and one block of ADR task. In one block of 1DR task, one of two directions (bull's eye mark) was rewarded throughout a block of experiments (40 trials), and the rewarded direction was changed in the next block. When a neuron was recorded in the $\mathrm{SNr}$, the rewarded direction was contralateral or ipsilateral to the recording side of the neuron, which will be referred to as the contralateral or ipsilateral rewarded block. The order of the blocks was randomized. In ADR, both directions were rewarded. For each trial in a block, the target was chosen pseudorandomly from two directions. For details, see Materials and Methods. contra, Contralateral; ipsi, ipsilateral.

and Wurtz, 1983a). Some of the recording sites were later confirmed histologically (see Fig. 10). We first selected SNr neurons that showed some task-related activity in ADR and then examined their spike activity using the standard test procedure (see Materials and Methods): ADR (one block) and 1DR (two blocks) (Fig. 1). For some neurons we also tested the four-direction version and found no qualitative differences in their activity patterns, and therefore we concentrate on the data based on the two-direction version. The positions of the targets were chosen such that one of them was located in the preferred location of the neuron (e.g., receptive field).

We recorded from $90 \mathrm{SNr}$ neurons that were related to ADR or 1DR. Their activity was associated with various task-related events. In this paper, we will focus on two dominant types: post-cue visual activity $(n=78)$ and pre-cue anticipatory activity $(n=74)$. Note that individual neurons often exhibited both types of activity.

\section{Characteristics of post-cue visual responses}

\section{Overview}

Post-cue responses of $\mathrm{SNr}$ neurons appeared as decreases or increases in activity from the high background level. The presence of post-cue activity was determined by comparing the firing rate in the post-cue test period with the firing rate in the control period for individual trials using paired $t$ test (see Materials and Methods). According to the analysis, 78 of 90 task-related $\mathrm{SNr}$ neurons showed post-cue activity. 
Figure 2. A sample $\mathrm{SNr}$ neuron showing decreasing direction and reward post-cue activity recorded from left side. The data obtained in two blocks of 1DR (left) and one block of ADR (right) are shown in columns. For each block (column), the cell discharge aligned on cue onset is shown separately for contralateral (top) and ipsilateral (bottom) directions as histogram/ raster displays aligned on the onsets of the fixation point ( fix on) and the cue stimulus (cue on). For each cue direction, the sequence of trials was from top to bottom. The rewarded direction is indicated by a bull's eye mark. Target eccentricity was $20^{\circ}$. The histograms have been smoothed by threepoint averaging based on data, with $10 \mathrm{msec}$ bin width. A horizontal line in each histogram indicates the mean discharge rate during the control period (1000 msec duration starting from $1300 \mathrm{msec}$ before fixation point onset). The decreasing response of the neuron to the contralateral cue was stronger when the cue indicated reward (top left). The neuron also showed decreasing pre-cue activity, but it was present in the 1DR-contralateral reward block and the ADR block.
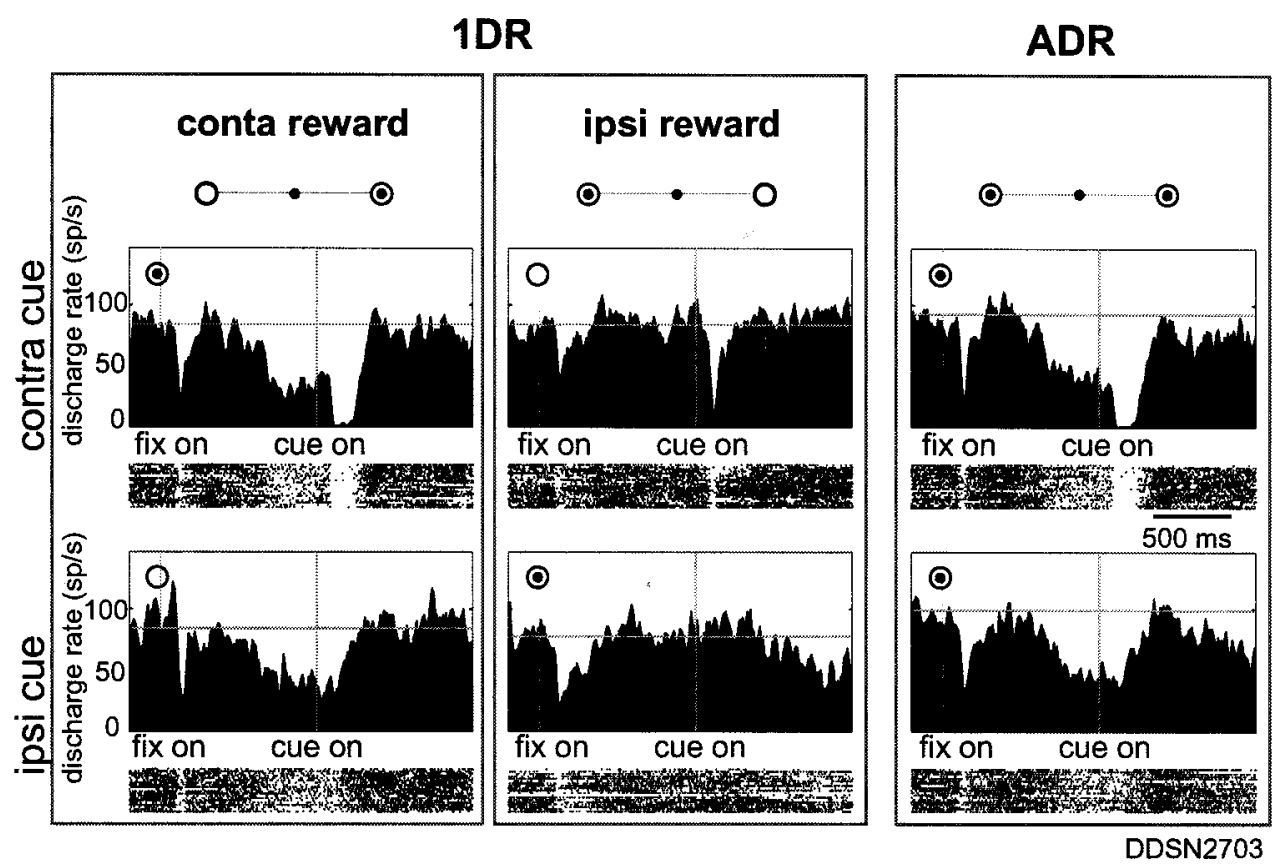

1DR
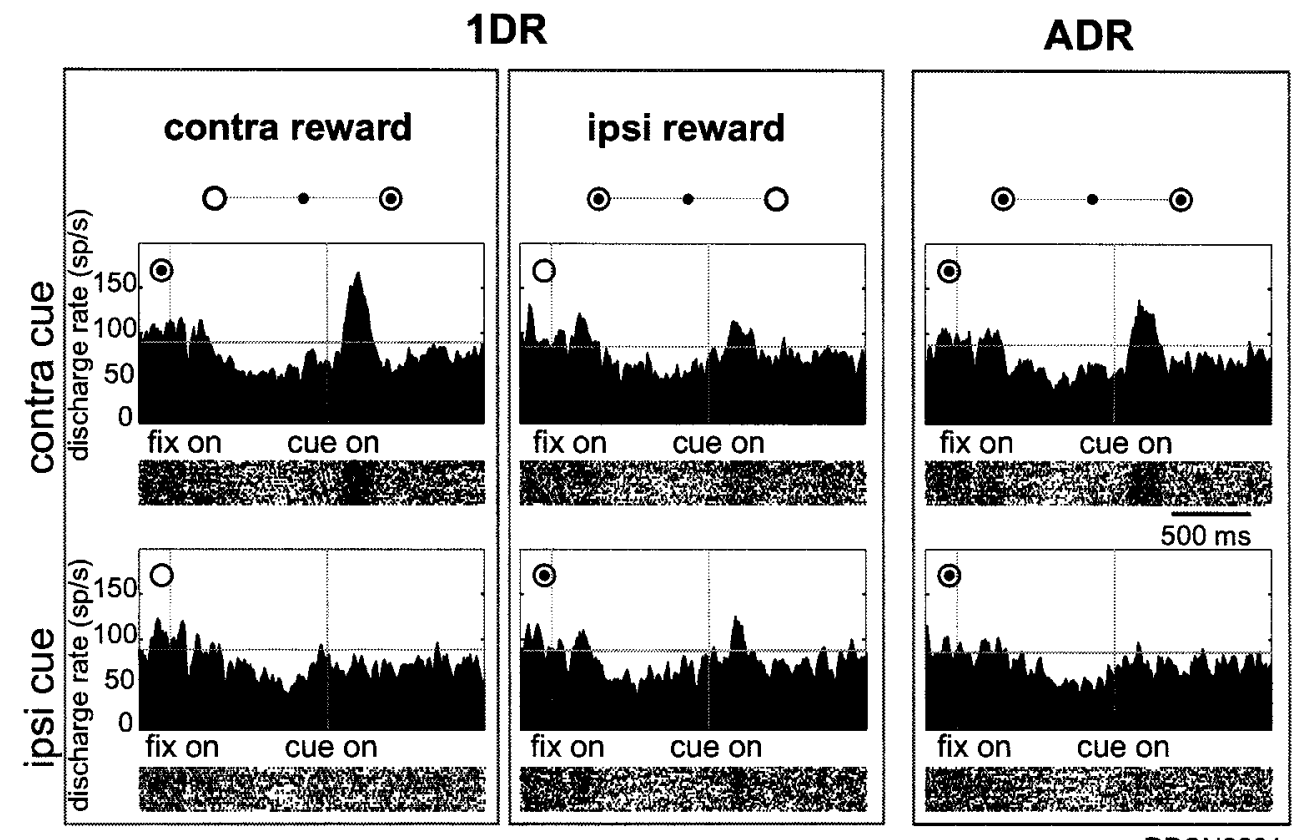

Figure 3. A sample SNr neuron showing increasing post-cue activity recorded from left side. The same format as in Figure 2. Target eccentricity was $20^{\circ}$.

DDSN3301

Using 1DR and ADR, we found that post-cue responses were determined by two factors: (1) retrospectively by the position of the visual cue (spatial factor) and (2) prospectively by the reward that would be given after the trial (reward factor). With respect to these factors, we classified $\mathrm{SNr}$ neurons into four types: (1) direction and reward (D\&R), (2) direction-only (D-only), (3) reward-only (R-only), and (4) nonmodulated (non-mod) (see Materials and Methods). Each group was further divided into decreasing (DEC) and increasing (INC) subtypes, such as D\&RDEC and D\&R-INC. We first show two examples of the D\&R type (Figs. 2, 3).

\section{Examples}

Figure 2 shows an example of decreasing post-cue response. The activity of the neuron is shown for two blocks of 1DR (left two columns) and one block of ADR (right column). The cue stimulus was presented randomly at one of two positions (one contralateral and the other ipsilateral) in each block, and the activity of the neuron is shown separately for the contralateral (top row) and ipsilateral (bottom row) stimuli. The neuron responded to the contralateral cue stimulus (top row), not to the ipsilateral cue (bottom row), by decreasing its activity in every block. Notably, the decreasing response showed positive reward modulation such that it was stronger in the rewarded condition (when the contralateral cue indicated an upcoming reward) (top left) than in the nonrewarded condition (top center) ( $t$ test; $p<0.0001)$. The results indicate that the cue direction was a primary determinant of the post-cue response of the neuron, and the reward condition modulated the re- 


\begin{tabular}{lccl}
\hline \multicolumn{4}{l}{ Table 1. Direction and reward modulations of post-cue activities } \\
& Decrease & Increase & Total \\
\hline D\&R & $20(26 \%)$ & $18(23 \%)$ & $38(49 \%)$ \\
D-only & $4(5 \%)$ & $8(10 \%)$ & $12(15 \%)$ \\
R-only & $7(9 \%)$ & $3(4 \%)$ & $10(13 \%)$ \\
Nonmodulated & $9(12 \%)$ & $9(12 \%)$ & $18(23 \%)$ \\
Total & $40(51 \%)$ & $38(49 \%)$ & $78(100 \%)$ \\
\hline
\end{tabular}

Table 2. Directional preference of post-cue activities

\begin{tabular}{lccl} 
& Decrease & Increase & Total \\
\hline Contralateral & $18(23 \%)$ & $19(24 \%)$ & $37(47 \%)$ \\
Ipsilateral & $6(8 \%)$ & $7(9 \%)$ & $13(17 \%)$ \\
Nonselective & $16(21 \%)$ & $12(16 \%)$ & $28(36 \%)$ \\
Total & $40(51 \%)$ & $38(49 \%)$ & $78(100 \%)$ \\
\hline
\end{tabular}

"Contralateral" indicates that the post-cue response was stronger to the contralateral than ipsilateral cues; "ipsilateral" indicates the opposite pattern.

sponse in a secondary manner. According to the criteria described in Materials and Methods, we classified this neuron as a D\&R-DEC type.

Figure 3 shows an example of increasing post-cue visual response. The neuron responded to the contralateral cue stimulus by increasing its activity. The increasing activity also showed positive reward modulation such that it was stronger in the rewarded condition (when the contralateral cue indicated an upcoming reward) (top left) than in the nonrewarded condition (top center) ( $t$ test; $p<0.0001)$. The neuron also showed a weak response to the ipsilateral cue when it indicated reward (bottom center). This neuron was thus classified as a D\&R-INC type.

\section{Classification of post-cue activity}

As illustrated for the sample neurons shown in Figures 2 and 3, we classified all $78 \mathrm{SNr}$ neurons that showed post-cue activity, and the results are shown in Tables 1 and 2. The analysis was done for three factors: (1) selectivity for cue direction (contralateral or ipsilateral), (2) selectivity for reward condition (present or absent), and (3) activity pattern (decrease or increase).

Table 1 shows that decreasing and increasing type neurons were similarly common (decrease, $n=40$; increase, $n=38$ ). Approximately half of the neurons $(n=34$; including neurons shown in Figs. 2, 3) were classified as D\&R type, that is, contingent on both the cue direction and the reward condition. Because our main interest in the present study was the interaction between the spatial and reward factors, we present several examples of D\&R type in Figure 4 for the direction factor and in Figure 6 for the reward factor.

Figure 4 shows, for each of the decreasing (left) and increasing types (right), the post-cue visual responses to the contralateral and ipsilateral cues for sample SNr neurons. The decreasing type responses were similar in pattern to each other, stronger or exclusive to the contralateral cue (thick line) for neurons 1-3 (Fig. 4, left, top three) and to the ipsilateral cue (thin line) for neuron 4 (Fig. 4, left, bottom). The population average of decreasing responses was much larger to the contralateral cue than to the ipsilateral cue (Fig. 5, bottom left). In contrast, the increasing type responses were variable in pattern, stronger to the contralateral cue for neurons 1-3 (Fig. 4, right, top three) and to the ipsilateral cue for neuron 4 (Fig. 4 , right, bottom). The contralateral domi-

\section{Decreasing type Increasing type}

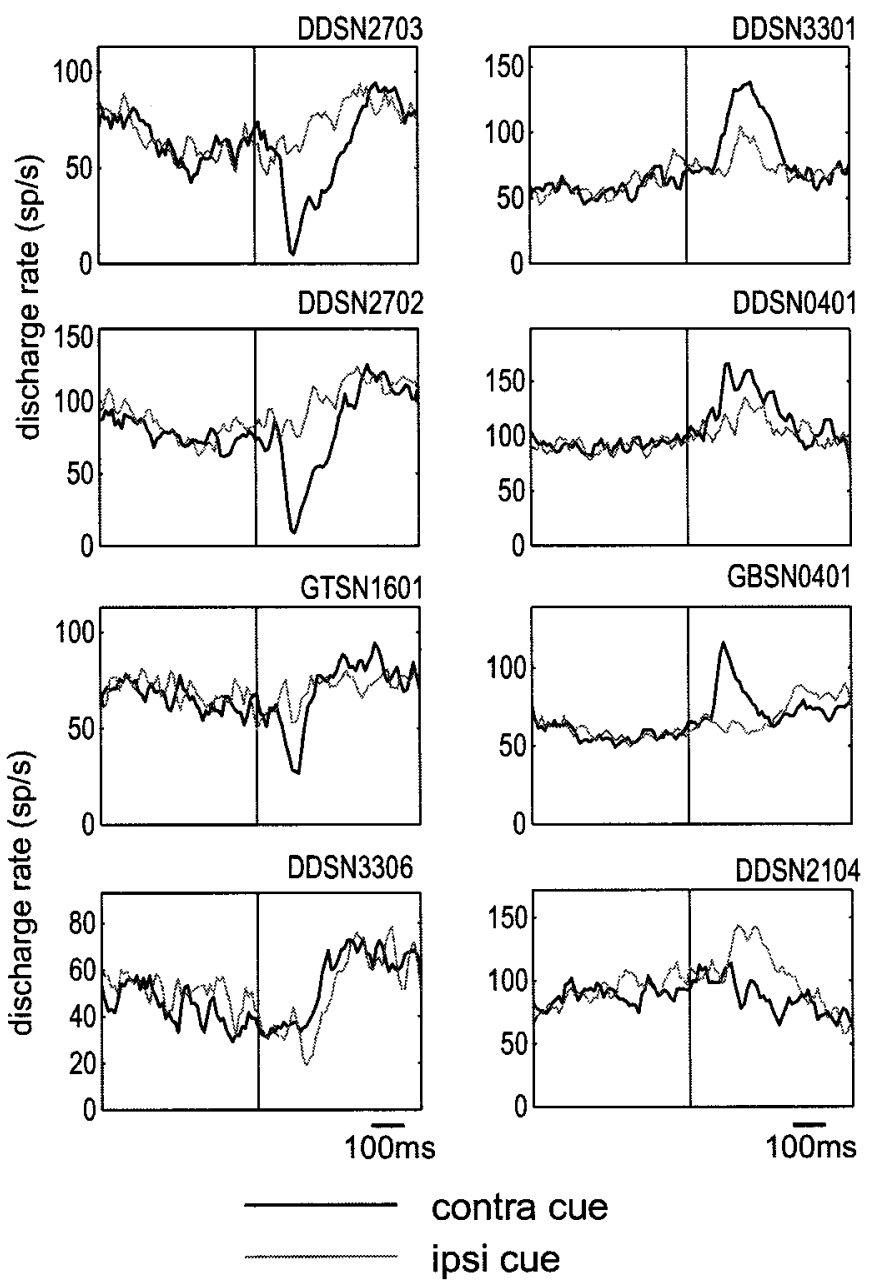

Figure 4. Spatial selectivity of post-cue visual responses of the decreasing type (left) and the increasing type (right). Four sample neurons are shown for each type. Histograms with thick and thin lines indicate the responses to the contralateral and ipsilateral cues, respectively, averaged for the two blocks of 1DR (rewarded and nonrewarded blocks). The histograms are aligned on the cue onset.

nance for the increasing type response observed as the population average was less clear than that for the decreasing type (Fig. 5, bottom right). Analysis for individual neurons showed that more than half of SNr neurons, either decreasing or increasing type, were direction selective, among which a majority preferred the contralateral direction (Table 2).

Figure 6 shows the reward effect, but only for the preferred direction of the neuron, because many neurons showed no response to the nonpreferred direction (as illustrated in Fig. 4) for the reward effect to be revealed. Decreasing type neurons (Fig. 6, left) invariably showed positive reward modulation: larger responses in the rewarded condition (thick line) than nonrewarded condition (thin line). Among increasing type neurons (Fig. 6, right), neuron 1 (top) showed positive reward modulation, whereas neurons 3 and 4 (bottom two) showed negative reward modulation. As a population, the decreasing response (Fig. 7, bottom left) was larger in the rewarded condition of 1DR than in the nonrewarded condition of 1DR or in ADR. In contrast, the increasing response (Fig. 7, bottom right) was not different be- 


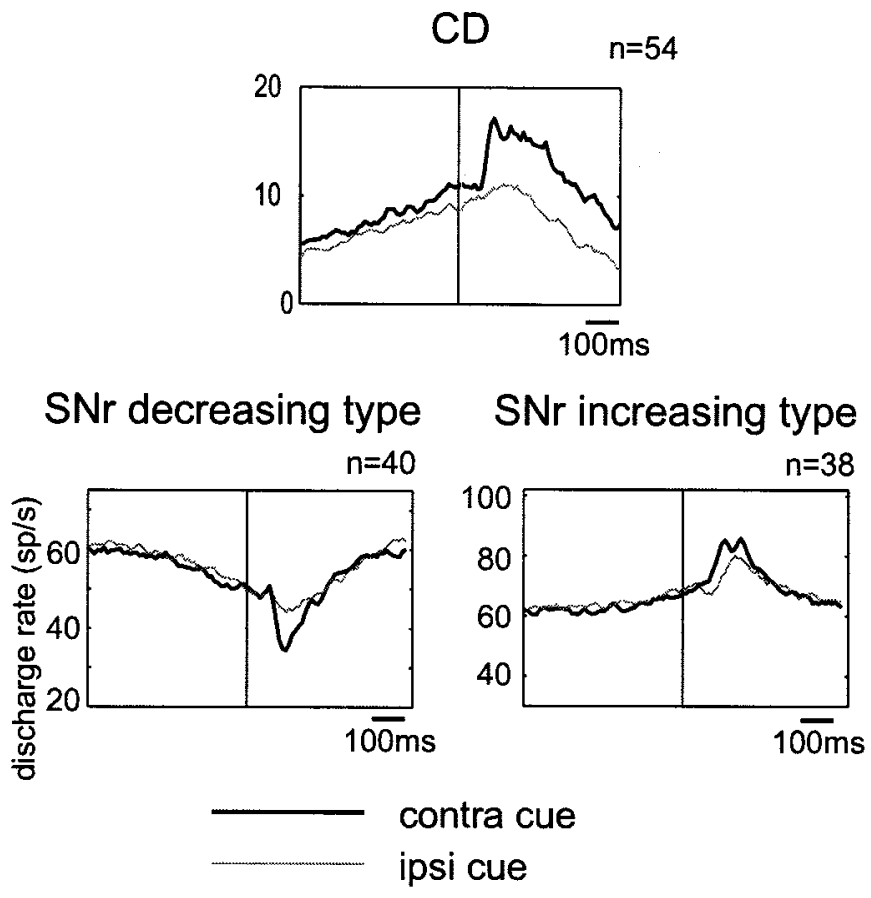

Figure 5. Spatial selectivity of post-cue responses in 1DR of SNr neurons, shown as population averages separately for the decreasing type (bottom left $;=40$ ) and the increasing type (bottom right; $n=38$ ). The population average of post-cue responses of CD projection neurons (top; $n=54$ ) [modified from Kawagoe et al. (1998)] is shown for comparison. Thick and thin lines indicate the responses to the contralateral and ipsilateral cues, respectively. Both the rewarded and nonrewarded trials are included.

tween the rewarded and nonrewarded conditions of 1DR, but was larger than in ADR. The analysis for individual neurons (Table 3) showed that more than half of $\mathrm{SNr}$ neurons were contingent on the reward condition. Positive reward modulation was dominant among decreasing type but not increasing type neurons. The population average also indicates that the decreasing post-cue response was preceded by the decreasing pre-cue activity, but preferentially in the rewarded condition (Fig. 7, bottom left).

\section{Spatial and reward indices}

To obtain a more general view, we defined and calculated the D-index and R-index for each neuron and plotted them in Figure 8, separately for the decreasing type (Fig. 8, left) and the increasing type (Fig. 8, right). Each type was further divided into four subtypes: D\&R, D-only, R-only, and non-mod. For the decreasing type (Fig. 8, left), most of the data points were in the top right quadrant, representing neurons that preferred the contralateral and rewarded cue. Preference was less clear for the increasing type (Fig. 8, right), except for some contralateral bias.

We found that R-index was statistically different among the eight neuron types (decreasing and increasing types taken together) (one-factor ANOVA; $p<0.001$ ), whereas D-index was not different $(p>0.05)$. A post hoc test (Tukey honestly significant difference) showed that R-index for D\&R-DEC type $(0.29 \pm 0.33$; mean $\pm \mathrm{SD})$ was higher than R-index for D\&R-INC type $(-0.06 \pm 0.26)(p<0.0001)$. We also examined whether the indices were significantly deviated from zero using one-sample $t$ test. The mean R-index was higher than zero for D\&R-DEC type $(p<0.001)$, but not for any other type. The mean D-index was higher than zero, but weakly, for D\&R-DEC type $(0.25 \pm 0.40$;

\section{Decreasing type Increasing type}

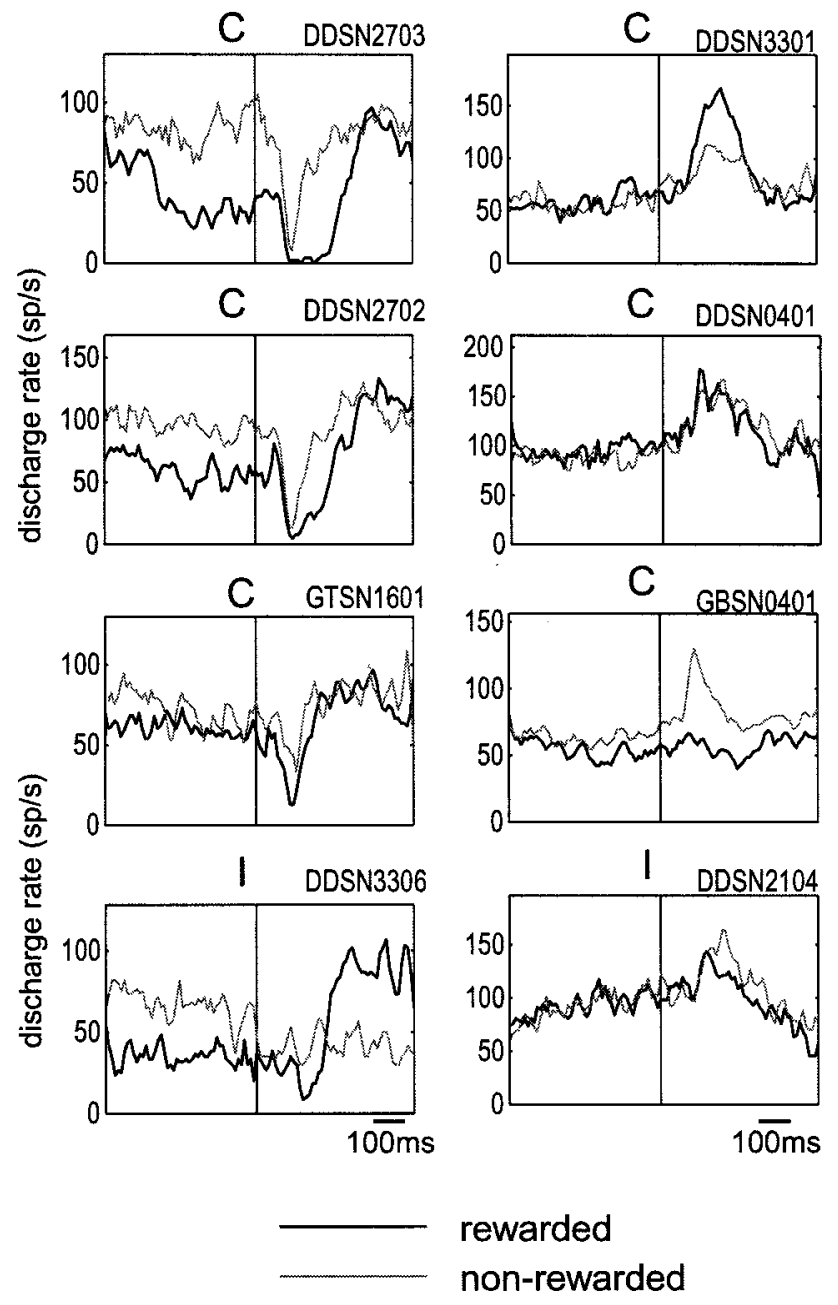

Figure 6. Modulation by predicted reward of post-cue visual responses of the decreasing type (left) and the increasing type (right). The same neurons as in Figure 4 are shown. Thick and thin lines indicate the post-cue responses in the rewarded and nonrewarded conditions, respectively. Shown are only the responses for the preferred direction of the neuron ( $C$, contralateral; $I$, ipsilateral), because there may be no response for the nonpreferred direction to show modulation.

$p<0.05)$ and D\&R-INC type $(0.10 \pm 0.19 ; p<0.05)$. Similar results were obtained for two types of neurons (decreasing and increasing types) after combining the four subtypes (ANOVA; $\mathrm{R}$-index, $p<0.01$; D-index, $p>0.05)$. The mean $\mathrm{R}$-index was higher than zero for the decreasing type (one-sample $t$ test; $p<$ $0.005)$ but not for the increasing type $(p>0.05)$. The mean D-index was higher than zero for the decreasing type $(p<0.005)$ and for the increasing type $(p<0.05)$. To summarize, there was the tendency that decreasing type neurons preferred the contralateral and rewarded cue, whereas increasing type neurons preferred also the contralateral cue but with no preference for rewarded or nonrewarded condition.

\section{Characteristics of pre-cue activity}

We found that many SNr neurons decreased $(n=51)$ or increased $(n=23)$ their activity before the cue stimulus was presented, which is what we call "pre-cue activity" (Table 4). Pre-cue and post-cue activity tended to be combined in single neurons: among 


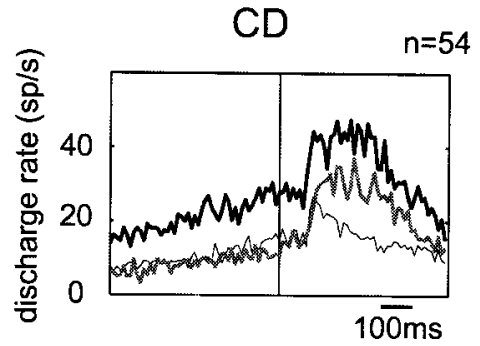

$\underset{n=40}{S N r}$ decreasing type $\quad$ SNr increasing type

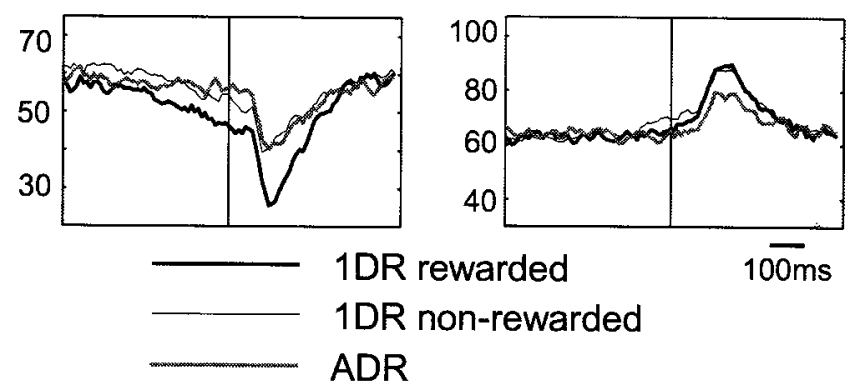

Figure 7. Modulation by predicted reward of post-cue visual responses of SNr neurons, shown as population averages separately for the decreasing type (bottom left) and the increasing type (bottom right). For comparison is shown the population average of post-cue responses of $\mathrm{CD}$ projection neurons (top) [modified from Kawagoe et al. (1998)]. Averaged histograms are shown for three reward conditions: contralateral-reward block of 1DR (thick line), ipsilateral-reward block of 1DR (thin line), and ADR (gray line). Both the contralateral and ipsilateral trials are included.

Table 3. Reward modulation of post-cue activities

\begin{tabular}{lcll} 
& Decrease & Increase & Total \\
\hline Positive & $23(29 \%)$ & $11(14 \%)$ & $34(44 \%)$ \\
Negative & $4(5 \%)$ & $10(13 \%)$ & $14(18 \%)$ \\
Nonmodulated & $13(17 \%)$ & $17(22 \%)$ & $30(38 \%)$ \\
Total & $40(51 \%)$ & $38(49 \%)$ & $78(100 \%)$
\end{tabular}

"Positive" indicates that the activity was enhanced when the cue indicated the upcoming reward; "negative" indicates that the activity was enhanced when the cue indicated no reward.

74 neurons with pre-cue activity and $77 \mathrm{SNr}$ neurons with postcue activity, 63 neurons showed both pre-cue and post-cue activity. Notably, the signs of these types of activity tended to be the same (e.g., pre-cue decrease followed by post-cue decrease) (Table 5). For example, the neuron presented in Figure 2 showed a gradual decrease before the cue onset, in addition to a phasic post-cue decrease.

Figure 9 shows examples of decreasing and increasing pre-cue activity in three reward conditions. Remarkably, the magnitude of pre-cue activity appears to be dependent on the reward condition. The decreasing pre-cue activity (Fig. 9, left) was usually larger in the contralateral-reward 1DR block than in the ipsilateral-reward 1DR block (e.g., first, second, and third neurons; also see Fig. 2). The decreasing pre-cue activity in ADR was weak (e.g., second and fourth neurons) or strong (e.g., first and fifth neurons), but usually did not exceed that in the contralateral-reward 1DR block. Among 51 neurons with decreasing pre-cue activity, the difference between the contralateral and ipsilateral reward conditions was statistically significant for 34 neurons ( $t$ test; $p<0.05$ ), and a majority (27) showed preference for contralateral reward (Table 4).
Decreasing type

Increasing type

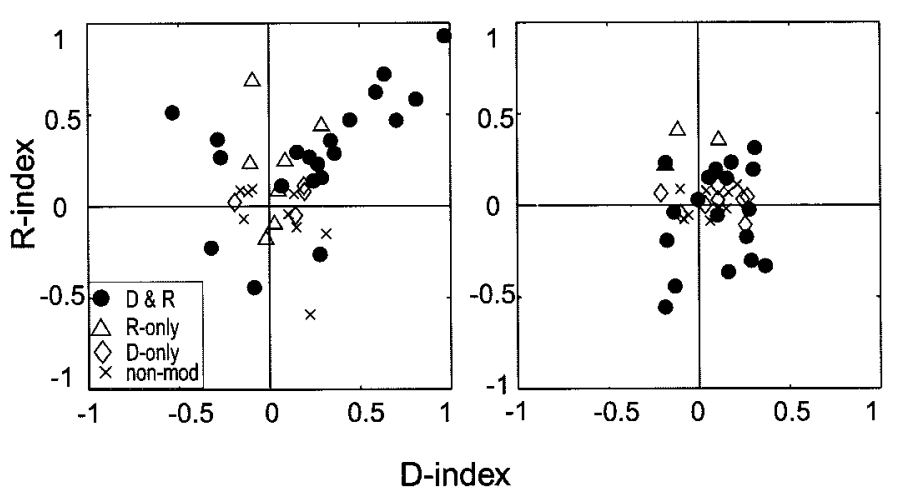

Figure 8. Spatial and reward-contingent modulations of post-cue activity: decreasing type (left) and increasing type (right). A single $\mathrm{SNr}$ neuron is represented by a dot indicating the D-index (abscissa) and the R-index (ordinate). The indices were calculated by the formulas shown in Materials and Methods. Indicated by different symbols are direction and reward type $(D \& R)$, direction-only type ( $D$-only), reward-only type (R-only), and nonmodulated type (non-mod).

Table 4. Preference of pre-cue activities for rewarded direction

\begin{tabular}{lccl} 
& Decrease & Increase & Total \\
\hline Contralateral & $27(36 \%)$ & $12(16 \%)$ & $39(53 \%)$ \\
Ipsilateral & $7(9 \%)$ & $5(7 \%)$ & $12(16 \%)$ \\
Nonselective & $17(23 \%)$ & $6(8 \%)$ & $23(31 \%)$ \\
Total & $51(69 \%)$ & $23(31 \%)$ & $74(100 \%)$
\end{tabular}

"Contralateral" indicates that the pre-cue activity was stronger when the contralateral cue indicated the upcoming reward than when the ipsilateral cue indicated the reward; "ipsilateral" indicates the opposite pattern.

Table 5. Combination of pre-cue and post-cue activities

\begin{tabular}{|c|c|c|c|c|}
\hline & \multicolumn{4}{|c|}{ Pre-cue } \\
\hline \multirow{4}{*}{$\begin{array}{l}\frac{0}{0} \\
\frac{1}{0} \\
0 \\
0\end{array}$} & & Decrease & Increase & Total \\
\hline & Decrease & $26(41 \%)$ & $5(8 \%)$ & $31(49 \%)$ \\
\hline & Increase & $16(25 \%)$ & $16(25 \%)$ & $32(51 \%)$ \\
\hline & Total & $42(67 \%)$ & $21(33 \%)$ & $63(100 \%)$ \\
\hline
\end{tabular}

The preference for reward direction was less clear and inconsistent for the increasing pre-cue activity (Fig. 9, right). It was sometimes larger in the ipsilateral-reward 1DR block (e.g., second neuron). The increasing pre-cue activity in ADR was usually weak. The difference was statistically significant for 17 of 23 neurons ( $t$ test; $p<0.05$ ), but the dominance pattern was less consistent (contralateral, 12; ipsilateral, 5) (Table 4).

The difference between the decreasing and increasing pre-cue activity was more evident as their population averages (Fig. 9, bottom). For the decreasing type (Fig. 9, bottom left), the pre-cue 


\section{Decreasing type (pre-cue)}
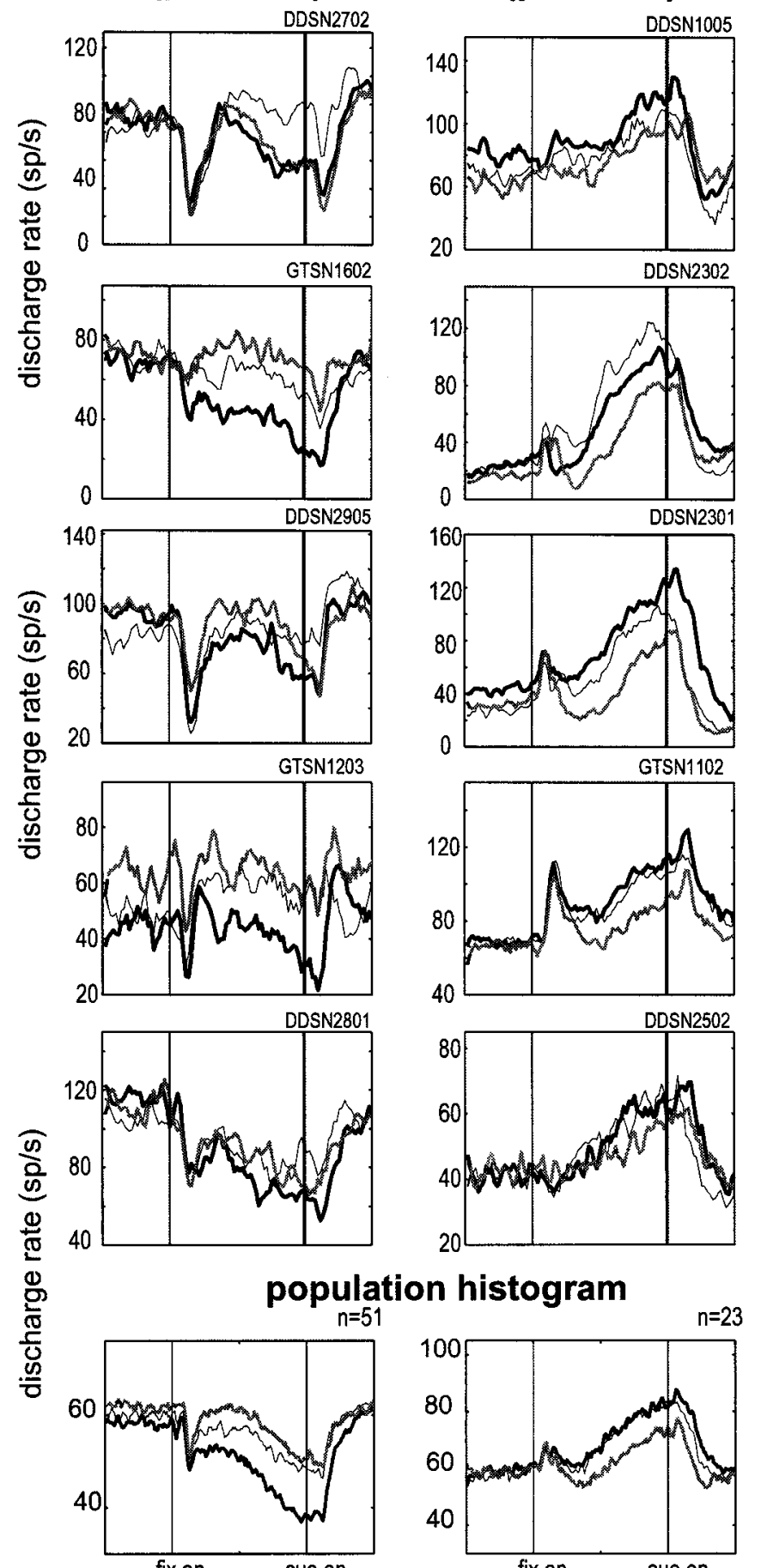

fix on

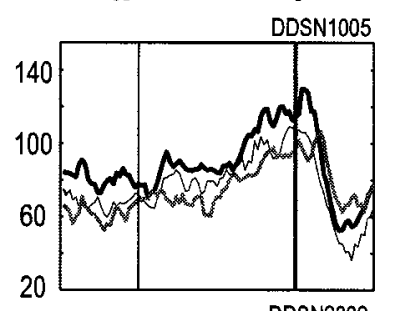

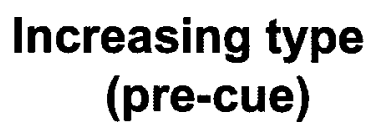

\section{as} coronal section that were directed to the $\mathrm{SNr}$ (Fig. 10A). In addition, there were two electrolytic marks along one of the electrode tracks. The lower mark corresponds to the location where the neuron shown in Figure 3 was recorded; it was in the dorsolateral part of the $\mathrm{SNr}$ (Fig. 10B).

With reference to such electrolytic lesions, we reconstructed the recording sites of task-related neurons recorded in one monkey (Fig. 10C-H). Most of them were mapped in the SNr. The decreasing and increasing types, together with some taskunrelated neurons, were intermingled in the SNr. Basically the same results were obtained from the other monkey.

\section{DISCUSSION}

On the basis of experiments using a position asymmetrically rewarded version of the memory-guided saccade task (1DR), we found that different groups of $\mathrm{SNr}$ neurons convey spatial and reward-related information in different combinations. These $\mathrm{SNr}$ neurons together would constitute an important mechanism for reward-oriented saccades.

\section{Decreasing and increasing responses of $\mathrm{SNr}$ neurons to visual cues}

$\mathrm{SNr}$ neurons showed decreasing and increasing changes in activity both in the post-cue visual period and in the pre-cue anticipatory period. For the post-cue activity, roughly equal numbers of $\mathrm{SNr}$ neurons showed decreasing and increasing changes. This observation differed from the original studies by Hikosaka and Wurtz (1983a) in which only decreasing responses were found and is in agreement with recent studies by Handel and Glimcher (1999, 2000). We confirmed histologically that both types of neurons were recorded inside the $\mathrm{SNr}$ (Fig. 10).

These changes in $\mathrm{SNr}$ neuronal activity may be caused by parallel and antagonistic pathways in the basal ganglia (Alexander and Crutcher, 1990) (Fig. 11). Thus, the decrease in SNr activity would be caused as a direct inhibition by striatal output neurons (Féger and Ohye, 1975), especially CD neurons (Hikosaka et al., 1993). The increase in SNr activity could be caused by multiple mechanisms, as an excitation by the subthalamic nucleus (STN) (Hammond et al., 1978; Nakanishi et al., 1987) or as a disinhibition by the globus pallidus external segment (GPe) (Smith and Bolam, 1990). The similarity in activity pattern among decreasing post-cue activity (Fig. 4, left) might be explained by the direct nature of the $\mathrm{CD}-\mathrm{SNr}$ inhibition, whereas the variation among increasing post-cue activity (Fig. 4, right) might be explained by the indirect and multiple pathways involving the GPe and STN.

decrease in activity was larger in the contralateral-reward 1DR
block than in the ipsilateral-reward 1DR block. In contrast, for the increasing type (Fig. 9, bottom right) the averaged pre-cue increase was nearly identical between the contralateral-reward activity in ADR block, either decreasing or increasing type, was smaller than that in any of the 1DR blocks.

Locations of SNr neurons
The position of the recording chamber was checked with magnetic resonance imaging before we started recording from a hemisphere. We also confirmed the recording sites histologically, shown in Figure 10. Several electrode tracks can be seen in this
Figure 9. Pre-cue activity of SNr neurons of the decreasing type (left) and the increasing type (right). Five sample neurons are shown for each type. Histograms are shown for three reward conditions: 1DR contralateralrewarded (thick line), 1DR ipsilateral-rewarded (thin line), and ADR (gray line). At bottom are shown the averages for all neurons that showed decreasing pre-cue activity (left; $n=51)$ and increasing pre-cue activity (right; $n=$ 23). Both contralateral-cue trials and ipsilateral-cue trials are included. 然 . . 
A

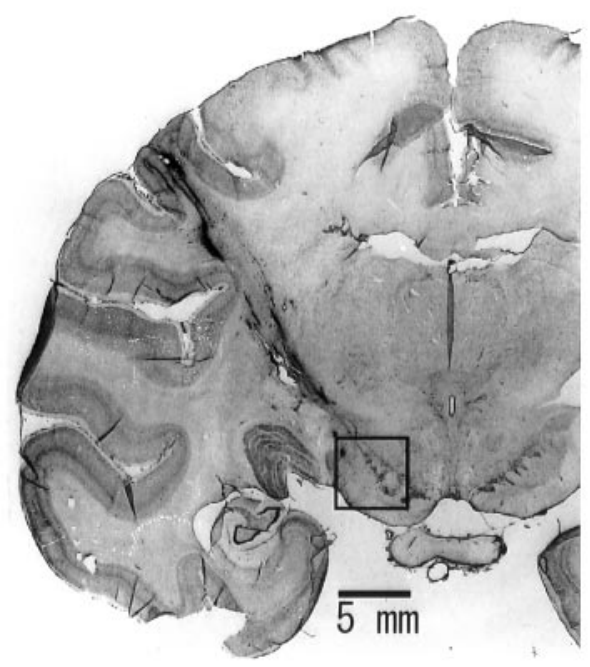

B
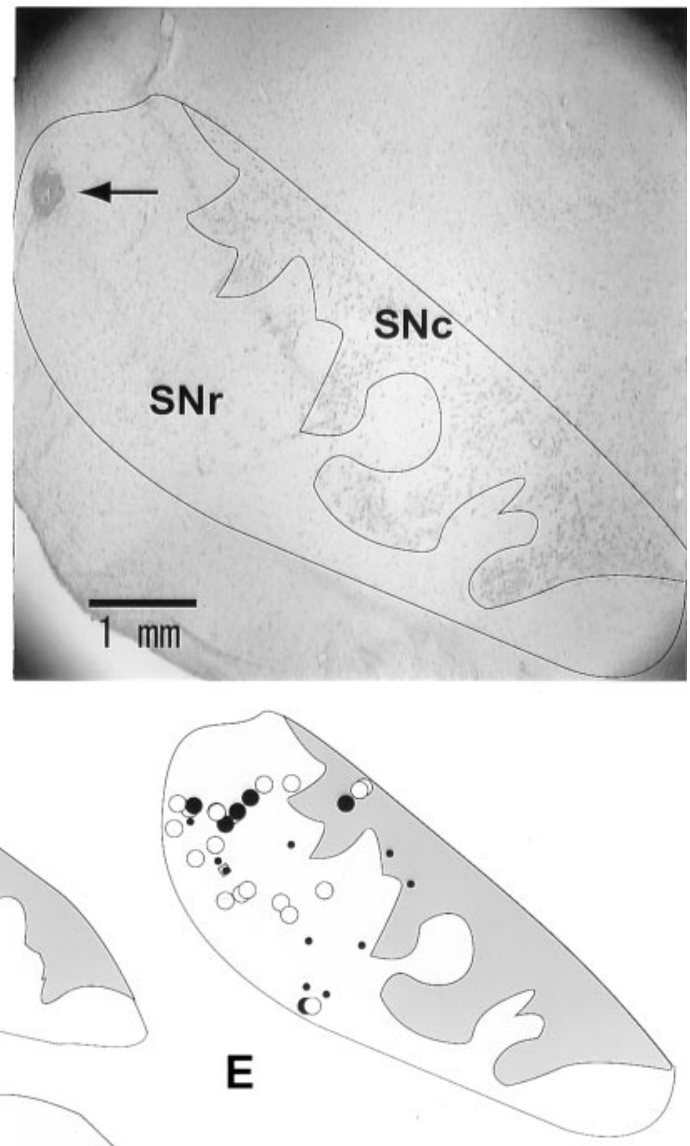

H

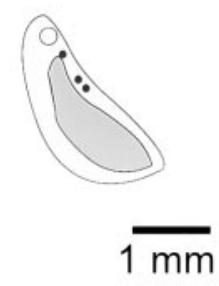

Figure 10. Recording sites of $\mathrm{SNr}$ neurons. $A$, A coronal histological section showing electrode tracks aimed at the SNr. In the enlarged photograph $(B)$ are indicated the $\mathrm{SNr}$ and the substantia nigra pars compacta $(S N c)$. An electrolytic mark (arrow) in the dorsolateral part of the $\mathrm{SNr}$ indicates the location where the neuron shown in Figure 3 was recorded. $\mathrm{C}-\mathrm{H}$, Recording sites of $\mathrm{SNr}$ neurons mapped on coronal histological sections from rostral $(C)$ to caudal $(H)$ with $0.5 \mathrm{~mm}$ intervals. The decreasing and increasing types (for their post-cue activity) are indicated by filled and open circles, respectively. Small dots indicate neurons that were not related to $1 \mathrm{DR}$ or ADR.

\section{Retrospective coding of visual signals and prospective coding of reward by SNr neurons}

The spatial selectivity of $\mathrm{SNr}$ neurons for visual, mnemonic, and saccadic activity has been well documented (Hikosaka and Wurtz, 1983a,b; Joseph and Boussaoud, 1985; Handel and Glimcher, 2000). Visual neurons usually have large receptive fields centered in the contralateral field (Hikosaka and Wurtz, 1983a; Handel and Glimcher, 1999). Consistent with these results, we found that most post-cue activity was larger to the contralateral than ipsilateral cues (Table 2). This was true for both the decreasing and increasing types. The contralateral dominance is expected by the fact that, physiologically, CD neurons usually show contralateral-dominant visual responses (Hikosaka et al., 1989b), and anatomically, the CD-SNr connections, both direct and indirect, are uncrossed (Williams and Faull, 1985).

We also found that the visual responses in $1 \mathrm{DR}$ were modu- lated prospectively by the presence or absence of reward. The modulation was positive (larger responses when reward was predicted) or negative (larger responses when reward was not predicted) compared with the responses in ADR. The positive reward modulation would not simply be caused by the presence of reward, because reward was given in every trial in ADR. Instead, it may depend on the uncertainty before cue onset, which could be called "reward prediction error" (Schultz, 1998). Alternatively, it could be caused by a difference in reward amount per trial (see Materials and Methods).

An important observation was the difference between the decreasing and increasing post-cue activity. The decreasing post-cue activity tended to show positive reward modulation, whereas the increasing post-cue activity showed positive modulations in approximately one-half of the neurons and negative modulations in the other half (Table 3, Fig. 8). This difference would require a specific relationship between the $\mathrm{CD}$ and the $\mathrm{SNr}$. 


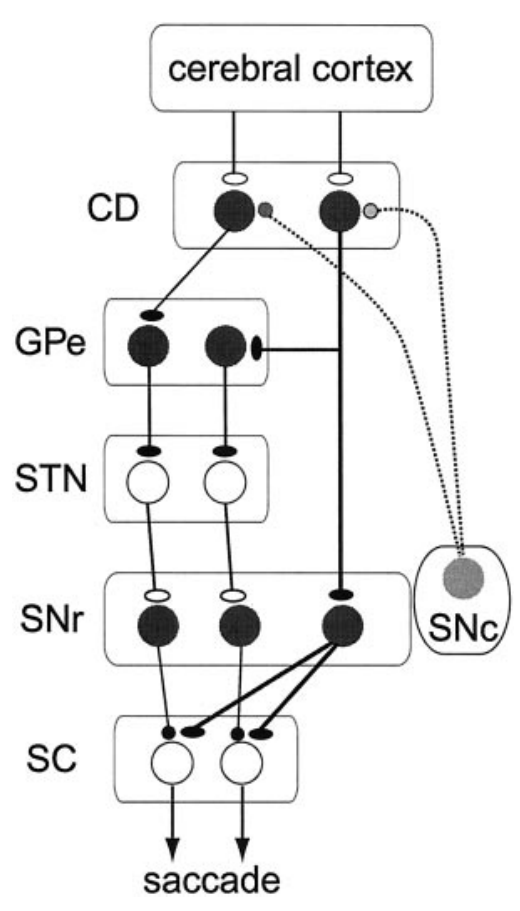

Figure 11. Hypothetical mechanisms for different types of SNr neuronal activity. $C D$, Caudate; $G P e$, globus pallidus external segment; $S T N$, subthalamic nucleus; $S N c$, substantia nigra pars compacta; $S C$, superior colliculus. Inhibitory and excitatory neurons are indicated by filled and open circles, respectively. Dopamine neurons in the SNc are shown in gray, indicating their modulatory roles for corticostriatal transmission. Different types of SNr neurons may receive different inputs from the CD: (1) decreasing type (one SNr neuron on the right), from the CD directly; and (2) increasing type (two SNr neurons on the left), from the CD indirectly through the GPe and the STN. CD neurons showing positive reward modulation (via D1 receptors, one $\mathrm{CD}$ neuron on the right) project to the $\mathrm{SNr}$ directly and in addition may have collateral connections to the GPe, whereas $\mathrm{CD}$ neurons showing negative reward modulation (via D2 receptors, one CD neuron on the left) project to the GPe only.

\section{Relation between caudate and substantia nigra pars reticulata}

According to a previous study from our laboratory using the same tasks (Kawagoe et al., 1998), many CD projection neurons showed increasing post-cue visual responses that were modulated by the predicted reward. The reward-contingent modulations were either positive or negative, the former being more common. Consequently, $\mathrm{SNr}$ neurons receiving direct inhibitory inputs from the CD would show post-cue decreases in activity, usually with positive reward modulations. This is exactly the pattern that we observed in $\mathrm{SNr}$ neurons of decreasing type. Indeed, the population activity of $\mathrm{SNr}$ neurons (decreasing type) and $\mathrm{CD}$ projection neurons were strikingly similar, except that their signs were reversed (Figs. 5, 7). The positive reward modulation may be mediated by dopamine D1 receptors on SNr-projecting CD neurons (Gerfen et al., 1990; Surmeier et al., 1996).

In contrast, increasing post-cue activity of SNr neurons would be caused by signals mediated by the indirect pathway (Fig. 11). It has been shown that GPe-projecting CD neurons express D2 receptors (Gerfen et al., 1990) and may mediate negative reward modulation (Wichmann and DeLong, 1996). We found, however, that increasing post-cue activity of $\mathrm{SNr}$ neurons showed both positive and negative reward modulations. An interesting possibility to explain the apparent discrepancy is that some CD neurons with positive reward modulation project to both the $\mathrm{SNr}$ and the GPe (Fig. 11), a scheme that would be supported by anatomical (Parent et al., 1995) and physiological (Surmeier et al., 1996) studies.

\section{Reward-oriented preparatory signals in the basal ganglia}

Pre-cue anticipatory activity was commonly observed among $\mathrm{SNr}$ neurons, as either a decrease or an increase in activity (Fig. 9). These two types were different in their selectivity. The decreasing pre-cue activity was selective for the rewarded direction. Typically, it was present only in the 1DR block when the contralateral side was rewarded. Such pre-cue activity cannot reflect simple prediction of a particular cue stimulus because the cue was presented randomly at one of the two positions. It would instead be related to the motivational state in which a particular position is associated with reward. Takikawa et al. (2002a) and Lauwereyns et al. (2002) have already reported very similar pre-cue activity in caudate projection neurons, although its sign was opposite (i.e., increase in activity). This again suggests the $\mathrm{CD}-\mathrm{SNr}$ inhibitory transmission for this information (Fig. 11).

In contrast, the increasing pre-cue activity overall had no such selectivity (although individual neurons were often selective). Considering its increasing nature, this type of pre-cue activity may be mediated by the indirect pathway from the $\mathrm{CD}$ to the $\mathrm{SNr}$, as suggested for the post-cue activity. It is not clear why the pre-cue activity has lost spatial selectivity during the signal transmission. Nonetheless, the increasing pre-cue activity did have selectivity in reward schedule such that it was generally stronger in 1DR than in ADR. This could be related to uncertainty of reward delivery or caused by the difference in reward amount. A similar selectivity was found for post-cue responses of presumed cholinergic interneurons in the CD (Shimo and Hikosaka, 2001). This raises the interesting possibility that the interneurons act preferentially on CD neurons projecting to the indirect pathway.

Functionally, the difference between the decreasing and increasing pre-cue activity is analogous with that observed for the post-cue activity. Similarly to the post-cue activity, the decreasing type was selective for reward (specifically rewarded direction), whereas the increasing type on the whole was not selective for reward. Furthermore, single SNr neurons tended to combine the decreasing pre-cue and post-cue activity or the increasing pre-cue and post-cue activity. These results suggest that pre-cue and post-cue activity are used for a common function, as discussed below.

\section{Functional significance: reward-oriented behavior}

Decreasing post-cue activity of $\mathrm{SNr}$ neurons occurred usually selectively to a contralateral cue. This would lead to a transient disinhibition of SC neurons so that a saccade is likely to occur toward the position of the contralateral cue. Important here is that this signal is larger when reward is expected. This means that when reward is expected, SC neurons would be more active, leading to an earlier and faster saccade (Takikawa et al., 2002b). Moreover, SNr neurons with decreasing post-cue activity often showed decreasing pre-cue activity and did so selectively when the contralateral side was rewarded (Figs. 2, 5, 7). In consequence, the post-cue decrease in activity was particularly large when the cue was presented on the contralateral side and it indicated reward (Fig. 2, top left). In short, the pre-cue and post-cue decreases, taken together, would act to facilitate a rewardoriented saccade.

What then could the function of increasing post-cue activity 
be? Increase in $\mathrm{SNr}$ neuronal activity should lead to inhibition of SC neurons and therefore suppression of contralateral saccades. This effect should be counteractive to the disinhibition by the decreasing type. However, they are critically different such that the post-cue decrease occurs preferentially in the rewarded condition, whereas the overall post-cue increase occurs nonselectively, although individual neurons are reward- or nonrewardselective (Fig. 6, bottom). In consequence, the $\mathrm{SNr}$ would facilitate reward-oriented saccades selectively while suppressing others.

\section{REFERENCES}

Alexander GE, Crutcher MD (1990) Functional architecture of basal ganglia circuits: neural substrates of parallel processing. Trends Neurosci 13:266-271.

Brown VJ, Desimone R, Mishkin M (1995) Responses of cells in the tail of the caudate nucleus during visual discrimination learning. J Neurophysiol 74:1083-1094.

DeLong MR, Georgopoulos AP (1981) Motor functions of the basal ganglia. In: The nervous system (Brooks VB, ed), pp 1017-1061. Bethesda, MD: American Physiological Society.

Féger J, Ohye C (1975) The unitary activity of the substantia nigra following stimulation of the striatum in the awake monkey. Brain Res 89:155-159.

Gerfen CR, Engber TM, Mahan LC, Susel Z, Chase TN, Monsma JFJ, Sibley DR (1990) D1 and D2 dopamine receptor-regulated gene expression of striatonigral and striatopallidal neurons. Science 250: $1429-1432$

Hammond C, Deniau JM, Rizk A, Féger J (1978) Electrophysiological demonstration of an excitatory subthalamonigral pathway in the rat. Brain Res 151:235-244.

Handel A, Glimcher PW (1999) Quantitative analysis of substantia nigra pars reticulata activity during a visually guided saccade task. J Neurophysiol 82:3458-3475.

Handel A, Glimcher PW (2000) Contextual modulation of substantia nigra pars reticulata neurons. J Neurophysiol 83:3042-3048.

Hikosaka O, Wurtz RH (1983a) Visual and oculomotor functions of monkey substantia nigra pars reticulata. I. Relation of visual and auditory responses to saccades. J Neurophysiol 49:1230-1253.

Hikosaka O, Wurtz RH (1983b) Visual and oculomotor functions of monkey substantia nigra pars reticulata. III. Memory-contingent visual and saccade responses. J Neurophysiol 49:1268-1284.

Hikosaka O, Wurtz RH (1989) The basal ganglia. In: The neurobiology of saccadic eye movements (Wurtz RH, Goldberg ME, eds), pp 257281. Amsterdam: Elsevier.

Hikosaka O, Sakamoto M, Usui S (1989a) Functional properties of monkey caudate neurons. I. Activities related to saccadic eye movements. J Neurophysiol 61:780-798.

Hikosaka O, Sakamoto M, Usui S (1989b) Functional properties of monkey caudate neurons. II. Visual and auditory responses. J Neurophysiol 61:799-813.

Hikosaka O, Sakamoto M, Usui S (1989c) Functional properties of monkey caudate neurons. III. Activities related to expectation of target and reward. J Neurophysiol 61:814-832.

Hikosaka O, Sakamoto M, Miyashita N (1993) Effects of caudate nucleus stimulation on substantia nigra cell activity in monkey. Exp Brain Res 95:457-472.

Hikosaka O, Takikawa Y, Kawagoe R (2000) Role of the basal ganglia in the control of purposive saccadic eye movements. Physiol Rev 80:953-978.

Houk JC, Adams JL, Barto A (1995) A model of how the basal ganglia generate and use neural signals that predict reinforcement. In: Models of information processing in the basal ganglia (Houk JC, Davis JL, Beiser DG, eds), pp 249-270. Cambridge, MA: MIT.

Joseph JP, Boussaoud D (1985) Role of the cat substantia nigra pars reticulata in eye and head movements. I. Neural activity. Exp Brain Res 57:286-296.

Judge SJ, Richmond BJ, Chu FC (1980) Implantation of magnetic search coils for measurement of eye position: an improved method. Vision Res 20:535-538

Kato M, Hikosaka O (1995) Function of the indirect pathway in the basal ganglia oculomotor system: visuo-oculomotor activities of external pallidum neurons. In: Age-related dopamine-deficient disorders (Segawa M, Nomura Y, eds), pp 178-187. Basal: Karger.

Kawagoe R, Takikawa Y, Hikosaka O (1998) Expectation of reward modulates cognitive signals in the basal ganglia. Nat Neurosci 1:411-416.

Kermadi I, Joseph JP (1995) Activity in the caudate nucleus of monkey during spatial sequencing. J Neurophysiol 74:911-933.

Kusama T, Mabuchi M (1970) Stereotaxic atlas of the brain of Macaca fuscata. Tokyo: University of Tokyo.

Lauwereyns J, Takikawa Y, Kawagoe R, Kobayashi S, Koizumi M, Coe B, Sakagami M, Hikosaka O (2002) Feature-based anticipation of cues that predict reward in monkey caudate nucleus. Neuron 33:463-473.

Matsumura M, Kojima J, Gardiner TW, Hikosaka O (1992) Visual and oculomotor functions of monkey subthalamic nucleus. J Neurophysiol 67:1615-1632.

Nakanishi H, Kita H, Kitai ST (1987) Intracellular study of rat substantia nigra pars reticulata neurons in an in vitro slice preparation: electrical membrane properties and response characteristics to subthalamic stimulation. Brain Res 437:45-55.

Nishino H, Ono T, Sasaki K, Fukuda M, Muramoto K-I (1984) Caudate unit activity during operant feeding behavior in monkeys and modulation by cooling prefrontal cortex. Behav Brain Res 11:21-33.

Parent A, Charara A, Pinault D (1995) Single striatofugal axons arborizing in both pallidal segments and in the substantia nigra in primates. Brain Res 698:280-284.

Robinson DA (1963) A method of measuring eye movement using a scleral search coil in a magnetic field. IEEE Trans Biomed Eng 10:137-145.

Rolls ET, Thorpe SJ, Maddison SP (1983) Responses of striatal neurons in the behaving monkey. I. Head of the caudate nucleus. Behav Brain Res 7:179-210.

Schultz W (1998) Predictive reward signal of dopamine neurons. J Neurophysiol 80:1-27.

Shimo Y, Hikosaka O (2001) Role of tonically active neurons in primate caudate in reward-oriented saccadic eye movement. J Neurosci 21:7804-7814.

Smith Y, Bolam JP (1990) The output neurones and the dopaminergic neurones of the substantia nigra receive a GABA-containing input from the globus pallidus in the rat. J Comp Neurol 296:47-64.

Surmeier DJ, Song W-J, Yan Z (1996) Coordinated expression of dopamine receptors in neostriatal medium spiny neurons. J Neurosci 16:6579-6591.

Takikawa Y, Kawagoe R, Hikosaka O (2002a) Reward-dependent spatial selectivity of anticipatory activity in monkey caudate neurons. J Neurophysiol 87:508-515.

Takikawa Y, Kawagoe R, Itoh H, Nakahara H, Hikosaka O (2002b) Modulation of saccadic eye movements by predicted reward outcome. Exp Brain Res 142:284-291.

Wichmann T, DeLong MR (1996) Functional and pathophysiological models of the basal ganglia. Curr Opin Neurobiol 6:751-758.

Williams MN, Faull RLM (1985) The striatonigral projection and nigrotectal neurons in the rat. A correlated light and electron microscopic study demonstrating a monosynaptic striatal input to identified nigrotectal neurons using a combined degeneration and horseradish peroxidase procedure. Neuroscience 14:991-1010.

Yoshida M, Precht W (1971) Monosynaptic inhibition of neurons in the substantia nigra by caudate-nigral fibers. Brain Res 32:225-228. 